\title{
Die Antragstellung für das europäische Zertifikat „Clinical Laboratory Geneticist" ist ab sofort möglich
}

Wie bereits berichtet ${ }^{1}$, verfolgt das European Board of Medical Genetics $(\text { EBMG })^{2}$ mit seiner Section Clinical Laboratory Geneticists seit 2012 das Ziel, den Titel "Clinical Laboratory Geneticist (CLG)" als eine EUweit anerkannte Fachqualifikation (as an EU-recognized specialist profession) in allen Europäischen Ländern einzuführen. Seit 2012 hat die Section CLG unter Vorsitz von Thomas Liehr die in den europäischen Ländern existierenden Weiterbildungsordnungen auf ihre Konformität mit dem Europäischen CLG-Curriculum geprüft. 15 Länder erfüllten bereits die Anforderungen, darunter auch Deutschland, Österreich und die Schweiz. Fachhumangenetiker/innen (GfH), Fachhumangenetiker/-innen (ÖGH) und die Spezialist/innen für medizinisch-genetische Analytik FAMH (SGMG) aus der Schweiz können nun das CLGZertifikat beantragen ${ }^{3}$.

\section{Die Antragstellung erfolgt in zwei Stufen:}

1. Pre-Registration: Die Antragsteller bekunden ihr Interesse an einer Anerkennung zum CLG und senden die nötigen Antragunterlagen $^{4}$ (abzurufen unter: https://www.eshg.org/587.0.html) bis spätestens 31.Oktober 2014 (ab 2015: 15. September) per Email an: PD Dr. Thomas Liehr (Thomas.Liehr@med.uni-jena.de) und Prof. Isabel Carreira (carreiraim@gmail.com).

\footnotetext{
${ }^{1}$ Ein ausführlicher Bericht dazu findet sich in: Liehr: European Board of Medical Genetics (EBMG) Section Clinical Laboratory Geneticists (CLG), medgen 3-2014, 406. Weitere Informationen auch in den Jahresberichten der Fachhumangenetiker-Kommission der GfH (medgen 1-2013, 27-28 und medgen 1-2014, 35-36).

2 Hierzu wurde 2012 das „European Board of Medical Genetics (EBMG)“ von der Europäischen Gesellschaft für Humangenetik (ESHG) gegründet, inzwischen ist es als, ,juristische Person“ anerkannt worden. Das EBMG ist rechtlich unabhängig von der ESHG, aber mit der ESHG assoziiert.

${ }^{3}$ Weitere Informationen und die für die Antragstellung benötigten Dokumente können unter https://www.eshg.org/407.0.html und https://www. eshg.org/587.0.html heruntergeladen werden.

${ }^{4}$ Eingereicht wird das unter den o.g. Adressen abrufbare Dokument $C$ als PDF mit einem eindeutigen Namen (z. B. NAME XYZ.150ct2014.pdf). Die Pre-Registration ist notwendig, weil in Zukunft nicht nur - wie momentan - Bewerbungen von CLG-Kandidaten aus den 15 europäischen Ländern mit EBMG-konformen Weiterbildungen eingereicht werden können, sondern aus 41 Ländern. Hier wird die Prüfung durch die EBMG komplexer ausfallen, insbesondere wird hier eine Vorprüfung der Unterlagen sinnvoll sein.
}

2. Registration: Sofern eine Vorprüfung ergibt, dass die Antragsteller die Voraussetzung für die Erlangung des CLG-Zertifikats erfüllen, werden sie gebeten, bis zum 15. Januar des Folgejahres ihre vollständigen Unterlagen einzureichen. Die hierfür benötigten Formulare und Angaben zu den einzureichenden Unterlagen finden Sie ebenfalls unter: https://www.eshg.org/587.0.html.

Die Mitteilung über Entscheidung zur Erteilung der Zertifikate erfolgt nach endgültiger Prüfung der Dokumente bis zum 1. April des Folgejahres.

WICHTIG: Die nationalen Weiterbildungsordnungen bleiben weiter in Kraft, Voraussetzung für den Erwerb des CLG-Zertifikats bleibt damit der Erwerb des nationalen Weiterbildungstitels.

\section{September 2014}

Thomas Eggermann

(Sprecher der

Fachhumangenetiker-

Kommission der GfH)

Martina Witsch-Baumgartner

(Sprecherin der

Fachhumangenetiker/in-

Kommission der ÖGH)
Thomas Liehr

(Vorsitzender der Section Clinical Laboratory Geneticists (CLG))

Wolfgang Berger (Co-Präsident FAMH der SGMG) 